the steelmaker builds up a personal knowledge and experience of the casting made by various alloys.

If the product of the furnace is required in the form of castings, the casting temperature must have its definite value. In this case its contours aro good, the shrinkage is a minimum, the structure is reason. ably strong and free-from blow holes. The author gives lists of the common defects associated with castings and also defects rising from incorrect treatment or application.

The engineer sometimes produces components which need to be heat-treated. The hardening shop has a great deal of responsibility, as it is the last stage of the work. The steelmaker goes to a great deal of trouble in determining the critical points of his alloys, which can be found from the heating and cooling curves, and from these ho computes the best temperatures for hardening. These temperatures must always bo used. The pyrometer must be poriodically inspected and calibrated. Useful informa. tion can often bo obtained from the microstructure as seen by microscopic examination of a representa. tive sample by means of a motallurgical microscope.

\title{
NUCLEOLI, SATELLITES AND SEX CHROMOSOMES
}

\author{
By Prof. R. Ruggles Gates, F.R.S.
}

$I^{N}$ recent years striking discoveries have been made regarding the origin and history of the nucleolus. It has como to bo recognized that in every cell of a plant or animal species thero is at least one pair of chromosomes which is specially concerned in pro. ducing this body. This pair of chromosomes may have either a satellite or a secondary constriction. In a satellited chromosome the oxact point of origin of the nucleolus appears to be the end of the chromosome proper, where the delicate thread or filament arises which attaches the satellite (a tiny globulo of chroma. tin) to the body of the chromosome. This locus has been called the nucleolar organizing body. In the telophase of mitosis each satellited chromosomo gives riso to a nucleolus, while in a chromosome with a secondary constriction the nucleolus arises at the constriction.

There are thus in the early telophase of mitosis as many nucleoli as there are chromosomes with satellites or secondary constrictions (the primary constric. tion being at the spindle fibre attachment). Some chromosomes with a secondary constriction, however, do not produce a nucleolus. By the following prophase the nuclcoli havo frequently all fused into one. By means of a now stain ${ }^{1}$, Feulgen and Light Green, which stains the chromatin (including the thread or filament) red and the nucleoli green, tho exact nature of tho attachment of nucleolar chromo. somes, whether at a satellite thread or a secondary constriction, can easily be determined. The number of chromosomes attached to the nucleolus in prophase is thus the same as the number which produce nucleoli in telophase. This relationship can generally be confirmed by an examination of the chromosomes in metaphase, when the number which have satellites or secondary constrictions can be determined, oxcept in the very smallest chromosomes.

In an ordinary diploid plant or animal species there is thus ono pair of chromosomes with satellites, or sometimes with secondary constrictions instead. This differenco secms to depend merely on whether the thin portion (thread) of the chromosome is near the end, to produce a satellite, or nearer the middlo of the chromosomo. In polyploid species the number of $S A T$-chromosomes and telophase nucleoli is corrospondingly increased. Thus a tetraploid will have four, and $\mathrm{a}$ hexaploid species such as the bread wheats will have six.
Some species, however, which have hithorto been regarded as ordinary diploids, have two pairs of nucleolar chromosomes. Sometimes ono of these pairs has satellites and the other pair secondary constrictions. Some of theso cases are clearly secondary tetraploids in which a now species or perhaps a now genus has arisen through a change in the basic number of chromosomes. Many such cases have been observed in connoxion with recent cytological work. The study of the nucleoli in a species has thus become a mothod of tracing changes in chromosomo number and how they have taken place, in other words, nuclear phylogeny.

As an example, rice may be cited. Cultivated rice, Oryza sativa, has twelve pairs of chromosomes. But many varieties have two pairs of nucleolar chromosomes, indicating that it is a secondary tetraploid or an amphidiploid. Further evidence is afforded by the secondary pairing of the chromosomes in the pollen mother cells. Such a pairing of chromosome pairs or bivalents with each other indicates a specific attraction, which is the result of homology.

The bivalent chromosomes arrange themselvesinrico so that the maximum association is into two groups of threo bivalents and three groups of two bivalents, $2(3)+3(2)$ or $A A A B B B C C D D E E$, thus making five groups of chromosomes having different homologies. That five is the basic number of chromosomes, from which the twelve in rice has been derived, was confirmed by showing (Ramanujarm ${ }^{2}$ ) that in certain genera related to Oryza, such as Zizania and Iygaeum, the chromosomes were in high multiples of five, whilo in other genera, such as Hygroryza, they were in multiples of twelve.

Many varioties of rice still have two pairs of $S A T \cdot c h r o m o s o m e s$, and so produce four nucleoli, confirming that they are secondary tetraploids. But it appears that it is only necessary for an organism to have one pair of $S A T$-chromosomes in order for the cells to function. In many varieties of rico only one pair is present. The other pair has been lost, probably by a mutational loss of a satellite from one of the four $S A T$-chromosomes and subsequent inbreeding. Several other wild species of Oryza, both in India and Africa, have doubled their chromosomes and have 24 pairs. They are thus secondary octoploids. This is an example of the way in which the origin and history of a genus can be traced by 
investigation of the behaviour of the chromosomes and especially of those which produce nucleoli. There is much other evidence that five is the fundamental chromosome number in the grasses, the family to which rice belongs.

Another recent example may be taken from the leguminous genus Cicer (Iyengar $\left.{ }^{3}\right) . \quad C$. arietinum has 16 chromosomes. In prophase four chromosomes are attached to the nucleolus and at meiosis the maximum association is one group of three bivalent chromo. somes, two groups of two and \& single bivalent, $1(3)+2(2)+1$ or $A A A B B C C D$. This indicates that four different (non-homologous) chromosomes was the original basic number. These observations confirm much other evidence (some of it unpublished) that four is the basic or original number of chromo. somes in the family of Leguminosæ, from which all other numbers in this family have been derived.

It is now well known that in groups of organisms as widely separated as insects and liverworts there are generally sex chromosomes, typically a large $X$ - and a small $Y$-chromosome. Moreover, theso bodies or portions of them are heteropyenotic, that is, they are condensed in prophase when the other chromosomes aro still in a diffuso condition. The heteropyenotic portions are believed to be genetically inert.

While the $X Y$ combination in insects, mammals and some other animals produces a male, in liverworts and mosses the $X Y$ pair are present in the sporophyte, the male gametophyte having $\mathbf{a} Y$ and the female gametophyto an $X$, in addition to the remainder of the haploid set of chromosomes.

Recent observations show that in many cases, both in insects and bryophytes, the $X$ - and $Y$-chromosomes are attached to the nucleolus, that is, they have a satellite or secondary constriction and aro concerned in producing the nucleolus to which they are attached. The possible significance of this widespread condition appears to have been overlooked. Kaufmann has shown that in somatic cells of male Drosophila melanogaster the $X$ (with a secondary constriction) and the $Y$ (with a satellite) are attached in prophase to the nucleolus which they produce. Heitz ${ }^{5}$ indicates the samo situation not only in $D$. melanogaster but also in $D$. funcbris, $D$. simulans, $D$. virilis and $D$. Hydei. By its heteropyenotic condition Heitz identifies the $X$-chromosome as attached to the nucleolus also in Scalophila unicornis and in two other geners of insects.

Thus it appears that in these insects the $X$ - and $Y$-chromosomes are generally nucleolar chromosomes, the former with a constriction and the latter with a satellite. An exception was found, however, by Kaufmann (1937) in Drosophila ananassae, in which the $X$ is not nucleolar, but a pair of autosomes (ordinary chromosomes) with satellites are attached to the nucleolus in the female. In the male, however, there are three chromosomes at tached to the nucleolus, the $S A T$-pair and the $Y$, which also has a satellite. Kaufmann suggests that this departure from the usual condition has been produced in this species by a translocation between the $X$-chromosome and an autosome.

From a study of the salivary gland nuclei of Droso. phila, Frolova finds the nucleolus attached by a branched chromatic thread to the chromo-centre, to which the ends of all the chromosomes are in turn attached. It seems probable that the sex chromosomes have here become detached from the nucleolus which they presumably produced, as in less specialized cells.
In liverworts, Lorbeer8 (1931) figures a large $X$. chromosome, evidently with a secondary constriction, attached to the nucleolus in Sphaerocarpus Donnellii, in the cells of the female thallus. In the cells of the male thallus there is a very small $Y$-chromosome and also an autosome with a satellite. In the spore mother cell there aro soven pairs of autosomes together with a large heteropycnotic $X$.chromosome attached to the nucleolus and the very small $Y$, also no doubt attached to the nucleolus when in its natural position. In $S$. europaeus the cells of the male thallus contain a $Y$ - and a $S A T$-chromosome, while in the female gametophyto an $X$ - and a $S A T$-chromosomo aro present. In Marchantia, both the $X$ and the $Y$ are figured attached to the nucleolus. Thus it appears that the sex chromosomes of the liverworts are nucleolus-producing, as in insects, but there are in some species also a pair of autosomes which produce nucleoli.

Allen' figures in Sphaerocarpus cristatus a heteropycnotic body which is part of the $X$-chromosome, attached to the nucleolus. In the liverworts Pallavicinia longispina and Calobryum rotundifolium, Tatuno $^{10}$ figures a large $X$-chromosome with a secondary constriction and a smaller $V$-shaped $Y$, both boing shown attached to the nucleolus in the spore mother cell of Pallavicinia. In Makinoa crispata, on the other hand, there are no heteropycnotic (sex) chromosomes, but the cells of the sporophyto show a pair of $S A T$-chromosomes. Tatuno ${ }^{11}$, in a further study, shows a large hetero. pycnotic $X$ and a small $Y$ in Pallavicinia Lyelli, Riccardia pinguis, R. blasioides, Pellia Nessiana and $P$. Fabbroniana. But in both species of Pellia a $S A T$-chromosomo is figured during mitosis in both the male spores (which have the $Y$ ) and the fomale spores (which have the $X$-chromosome).

In mosses, similar conditions hold. Shimotomai and Koyama12 (1932) show in the female gametophyte of Pogonatum inflexum a large heteropycnotic $X$ chromosomo attached to the nucleolus, a small $Y$-chromosome attached in the nucleoli of the male gametophyte, and both attached to the nucleolus in the cells of the seta (sporophyte). Shimotomai and Kimura ${ }^{13}$ show similar conditions in Polytrichum and Ceratodon.

Thus it appears that both in insects and bryophytes tho sex chromosomes talie part, with or without a pair of autosomes, in forming the nucleoli. In diccious flowering plants the relation of the $X$. and $Y$-chromosomes to nucleolar production is not yet known. By the use of the new staining method already mentioned, it will be possible to trace their history fully in flowering plants and also to throw further light on the behaviour of the sex chromosomes in other organisms.

1 Semmens, C. S., and Bhaduri, F. T., Stain Tech., 14, 1-5 (1939).

Ramanujam, S., Ann. Bot., N.S., 2, 107-125 (1938).

- Iyengar, N. K., Ann. Bot., N.S., 3 (1939).

- Kaufmann, B. P., J. Morrh., 56, 125-154 (1934).

s Ifeitz, E., Z. Zellforsch, u. MIikr. Anat., 20, 237-287 (1933); 19, $720-742$ (1933).

- Kaufmann, B. P., Cytologia, Fujil Jub. Vol. 1043-1055 (1937).

- Frolova, S. L., Biol. J. (Russian), 5, 271-292 (1936).

- Lorbeer, G., Jahtb. uiss. Bot., 80, 567-818 (1934).

- Allen. C. E.. Bot. Gaz., 97, 846-853 (1936).

10 Tatuno, S., J. Sci. Hiroshima Unir., 1. 165-182 (1933).

11 Tatuno, S., J. Sci. Miroshima Unit., 3, 1-9 (1936). See also Bot. Mag. Tolyo., 51-53.

18 Shimotomai, N., and Koyama, J. Sci. Hiroshima Unic., Ser. I., Div. 2, 1. 95-101 (1932).

${ }^{13}$ Shimotomai, N., and Kimura, K.. Z. Abst. ${ }_{-}$. Veretb., 72, 307-312 (1936). 